\title{
Foliar Amino Acids Sprays on Lettuce (Lactuca sativa L.) Biomass Accumulation
}

\author{
M. Salinas ${ }^{1}$, E. Gandolfo ${ }^{1}$, G. Hakim ${ }^{1}$, E. Giardina ${ }^{1}$ and A. Di Benedetto ${ }^{1,2} *$ \\ ${ }^{1}$ Faculty of Agronomy, University of Buenos Aires, San Martín Avenue 4453 (C1417DSE), \\ Buenos Aires, Argentina \\ ${ }^{2}$ Faculty of Agricultural Sciences, National University of Mar del Plata, Route 226, km. 73.5 \\ (B7620ZAA), Balcarce, Province of Buenos Aires, Argentina \\ *Corresponding author:
}

\section{A B S T R A C T}

\begin{tabular}{|l|}
\hline Ke y w or d s \\
Bio stimulants, \\
Vegetables, Abiotic \\
stress
\end{tabular}

Nitrogen has been associated to lettuce fresh weight accumulation. In this way, different bio stimulants formulations containing amino acids have been commercially offered although the yield increase has not been properly supported. The aim of this work was a) to quantify the changes in lettuce fresh weight accumulation and the physiological mechanism involved in plants sprayed with different amino acid solutions and (b) to characterize the responses to root restrictions under nursery with non-limited nitrogen supply. Our results showed that a foliar spray with different amino acids each alone or combined can change positively or negatively lettuce fresh-dry weight, total leaf area and photo assimilate partitioning under a nursery abiotic stress related to plug cell size. Although, exogenous amino acids supply can be suggested as signalling molecules, the presence of limited plug cell volume (288-plug cell tray) would be demanded for the use of more than a single one. On the other hand, although additive and antagonistic effects have been suggested, conclusive results needs for further experiments.

\section{Introduction}

Both availability and uptake of nitrogen are considered as the major factors affecting plant growth. Nitrogen acquisition by plant roots occurs to a variety of inorganic (nitrate, ammonium and urea) and organic (amino acids and peptides) nitrogen sources (Andrews et al., 2013). Nitrogen metabolism exerts a strong effects on both photo assimilates fixation and partition between roots and shoots (Poorter et al., 2012). Nitrate reductase, nitrite reductases and the glutamate synthase cycle catalyzed organic nitrogen assimilation. The Glutamate occupies a central position in amino acid metabolism in plants (Filleur et al., 2005; Schachtmanand Shin, 2007).

Main amino acids (glutamate) have been correlated with nitrogen acquisition while minor amino acids such as arginine, phenylalanine, tyrosine and valine have been correlated with photo assimilates storage (Foyer et al., 2003). At the same time, 
arginine and proline have been indicated as precursors of polyamides associated to the plant response towards abiotic stresses (Forde and Lea, 2007; Biancucci et al., 2015) and functioned as signaling molecules (Häusler $e t$ al., 2014).

On the other hand, Stitt et al., (2002) showed that addition of ammonium, glutamine, or glutamate, did not lead to a significant increase in the minor amino acids levels, indicating that flux into the amino acid biosynthesis pathways is not limited by the availability of glutamate in main plant metabolism, at least in plants where the nitrogen supplies is not limiting.

Previous reports indicate that exogenous amino acids supply increased growth and yield to different horticultural species such as lettuce (Polo et al., 2006), celery (Shehata et al., 2011), spinach (Kunicki et al., 2010), leaf radish (Liu et al., 2008), pepper (Haghighi and Barzegar, 2017) and squash (El-Aal et al., 2010) but, the precise physiological mechanism involved in these responses remained unsolved.

Lettuce is a major crop grown worldwide (Stagnari et al., 2015; Shehata et al., 2016). Lettuce yields increase according to photosynthetic light increase (He et al., 2011) as a response to a nitrate reductase increase (Stagnari et al., 2015). In the same way, lettuce yields are positively related to nitrogen supply (Linker and Johnson-Rutzke, 2005).However, lettuce is also prone to accumulation of nitrates, which are recognized to be dangerous to human health (Fewtrell, 2004).

Transplanting has been thoroughly replacing vegetables direct seeded since plug trays have been commercially offered in spite of the root restriction syndrome related to the limited plug cell volume (Araki et al., 2007; Di
Benedetto, 2011; Coro et al., 2014; Sanchez Andonova et al., 2014; Di Matteo et al., 2015).

The objective of this work was to analyze lettuce growth changes in the presence of different root restrictions degree by the use of different plug cell volumes but sprayed with amino acids solutions under the hypothesis that they would play a role as abiotic stress alleviators.

\section{Materials and Methods}

The experiment was carried out in the Faculty of Agronomy campus, University of Buenos Aires, Argentina (3435' 59' 'S, 58 $22^{\circ}$ ' $23^{\prime}$ 'W) between October $30^{\text {th }} 2017$ and February $26^{\text {th }} 2018$.

To reach proposed objectives, lettuce (Lactuca sativa L.) Crimor INTA seeds were grown in $50-\left(55.7 \mathrm{~cm}^{3}\right.$ cell $\left.^{-1}\right)$ and $288-\left(6.18 \mathrm{~cm}^{3}\right.$ cell $\left.^{-1}\right)$ plug trays in a Klasmann411® medium (Klasmann-Deilmann, GmbH, Germany) for 35 days. When seedlings reached the transplant stage, they were transplanted into 3 liters pots filled with a Sphagnum maguellanicum-river waste-perlite (40-40-20, $\mathrm{v} / \mathrm{v} / \mathrm{v})$ medium. At the beginning of the experiments total porosity (\%), air-filled porosity $(\%)$, container capacity $(\%)$ and bulk density $\left(\mathrm{g} \mathrm{cm}^{-3}\right)$ were $63.50,17.06,10.06$ and 0.35 respectively. Weeds were manually removed.

Eleventh solutions (100 $\mathrm{mg} \mathrm{L}^{-1}$ ) containing an equal amino acids number (glutamic acid, aspartic acid, alanine, cysteine, glycine, leucine, lysine, methionine, threonine, tryptophan and valine), a control without treatment and a solution containing all amino acids (Mix) (100 $\mathrm{mg} \mathrm{L}^{-1}$ ) were tested. Leaves were sprayed at sunset when the first true leaf pair was developed. Plants were irrigated as needed with high quality tap water $(\mathrm{pH}: 6.64$ 
and electrical conductivity of $0.486 \mathrm{dS} \mathrm{m}^{-1}$ ) using intermittent overhead mist to compensate evapotranspiration loses. Growing media was weekly fertilized with 1:1:1:1 $(\mathrm{v} / \mathrm{v} / \mathrm{v} / \mathrm{v}) \mathrm{N}: \mathrm{P}: \mathrm{K}: \mathrm{Ca}$ through the overhead water irrigation (Stage 2: $50 \mathrm{mg} \mathrm{L}^{-1} \mathrm{~N}$; Stage 3-4:100 $\mathrm{mg} \mathrm{L}^{-1} \mathrm{~N}$; pot: $200 \mathrm{mg} \mathrm{L}^{-1} \mathrm{~N}$ ) was used.

Daily mean temperatures $\left(18.05\right.$ to $\left.24.86{ }^{\circ} \mathrm{C}\right)$ and daily photosynthetic active radiation $(6.83$ to11.16 mole photons $\mathrm{m}^{-2}$ day $^{-1}$ ) for the experiment were recorded with a $\mathrm{HOBO}$ sensor (H08-004-02) (Onset Computer Corporation, MA, USA) connected to a HOBO H8 data logger. The plants were arranged at a density of six plantsm ${ }^{-2}$, which avoided mutual shading.

Plants were harvested at the transplant stage and at 20,40, 60, 80, 100 and 120 days from transplanting. Roots were washed and root, stem and leaf fresh weights (FW) were recorded. Dry weights (DW) were obtained after drying roots, stems and leaves to constant weight at $80^{\circ} \mathrm{C}$ for 96 hours. The number of leaves was recorded, and each leaf area was determined using the Image ${ }^{\circledR}$ (Image Processing and Analysis in Java) software.

The relative rate of leaf area expansion (RLAE) was calculated as the slope of the regression of the natural logarithm (ln) of total leaf area versus time (in days). The rate of leaf appearance (RLA) was calculated as the slope of the number of fully expanded leaves versus time (in weeks). The relative growth rate (RGR) was calculated as the slope of the regression of the natural logarithm (ln) of whole plant DW versus time (in days).

Mean net assimilation rate (NAR) was calculated as follows:

$$
N A R=\frac{k_{w} W_{0} e^{k_{w} t}}{A_{0} e^{k_{a} t}}
$$

where $\mathrm{W}_{0}$ : extrapolated value of total DW $(\mathrm{g})$ at time zero; $\mathrm{k}_{\mathrm{w}}$ : RGR $\left(\mathrm{g} \mathrm{g}^{-1} \mathrm{day}^{-1}\right) ; \mathrm{A}_{0}$ : extrapolated value of leaf area $\left(\mathrm{cm}^{2}\right)$ at time zero; $\mathrm{k}_{\mathrm{a}}: \operatorname{RLAE}\left(\mathrm{cm} \mathrm{cm}^{-2}\right.$ day $\left.^{-1}\right)$; t: time (days) at the midpoint of the experimental period and e: base of the ln.

Mean and leaf area ratio (LAR) was calculated as follows:

$$
L A R=\frac{k_{w}}{N A R}
$$

The specific leaf area (SLA) and the leaf weight ratio (LWR) on a dry weight (DW) basis were calculated as the ratio of leaf area: leaf DW and the ratio of leaf DW: total DW respectively between the transplant stage and the end of the experiment.

The allometric coefficients between root and shoot were calculated as the slope $(\beta)$ of the straight-line regression of the $\ln$ of the root DW versus the $\ln$ of the shoot DW (ln root DW $=a+b x \ln$ shoot DW).The Root: Shoot ratio (at the end of the experiment) was performed as well.

We used a complete aleatory design. Data were subjected to one-way analysis of variance and means were separated by Tukey's test $(\mathrm{P}<0.05)$; STATISTICA 8 (StatSoft) software was used. Least significant differences (LSD) values were calculated. Slopes from straight-line regressions of RLAE, RLAE, RGR and allometric value were tested using the SMATR package (Warton et al., 2012).

\section{Results and Discussion}

Significant statistical differences in posttransplant fresh weight accumulation have been found in lettuce plants sprayed with 12 amino acids solutions during nursery. Plants grown in 50 -cell tray ${ }^{-1}$ showed higher values than those 
from 288-cell tray ${ }^{-1}$. At the end of the experiment (120 days from sowing) 50-cell plug tray ${ }^{-1}$ plants showed no significant increases in most of the amino acids solutions sprayed and controls but, significant decreases in fresh weight in some amino acids solutions tested (Mix, glutamic acid, cysteine, glycine, leucine, valine). On the other hand, lettuce seedlings grown in 288-cell plug tray ${ }^{-1}$ showed a significant increase related to controls when a combined solution (Mix) was applied and higher response heterogeneity between the rest single-applied amino acids (Table 1).

Under a relatively low plug cell volume abiotic stress, this is, in plants grown in 50-cell plug tray $^{-1}$, total leaf area differences between controls and amino acids ones were significant in the latter two harvest time (data not shown). In plants grown at 50-cell plug tray ${ }^{-1}$, the higher total leaf area was found in alanine-sprayed plants; on the contrary, plants sprayed with aspartic acid, glycine and leucine showed the lowest values even to control plants. However, when seedlings were grown in 288-cell plug trays during nursery, the higher total leaf area was found in the Mix solution following by control plants (Table 2).

The higher control RLAE and RLA values were found in plants from 50-cells plug tray ${ }^{-1}$, while an inverse SLA results was found. The Mix solution which contained the thirteen amino acids tested, increased RLAE and RLA in plants grown in 288-cells plug tray ${ }^{-1}$. In 50cells plants, all amino acids sprayed increased RLAE on control and Mix plants, although showed a heterogenic response from RLA (Table 2).

Plants grown in 50-cells plug tray ${ }^{-1}$ and sprayed with glycine and leucine, showed the lower shoot dry matter accumulation while the lower root dry weight values was found in control plants (Figure 1A). Plants grown in 288-cells plug tray ${ }^{-1}$, the higher shoot dry weight was found in the Mix, leucine, lysine, threonine and tryptophan sprayed plants. Once again, the lowest root dry weight was found in control plants (Figure 1B). Anyway, plants from 50cells plug tray ${ }^{-1}$ showed even the highest both shoot and root dry weight than those from 288cells plug tray ${ }^{-1}$.

The higher both RGR and NAR were found in plants from 50-cells than in those from 288plug tray ${ }^{-1}$ while an inverse LAR response was found. RGR did not change significantly from 50-cells grown plants amino acid-sprayed while the Mix treatment increased RGR in 288cells grown plants. All of the twelve amino acids solutions increased 50-cell grown plants NAR but only the Mix treatment increased 288-cell grown plants NAR. LAR showed only significant minor changes from 50-cell plug tray $^{-1}$ and no significant differences in those from 288-cell plug tray ${ }^{-1}$ (Table 3).

When NAR and LAR values were plotted together, positive relationships between NAR, LAR and RGR were found (Figure 2). Lettuce plants from 50-cell plug tray ${ }^{-1}$ showed higher determination coefficients $\left(\mathrm{r}^{2}\right)$ than those from 288-cell plug trays.

Higher root: shoot ratios were found in plants from 50-cells plug tray ${ }^{-1}$ than those from 288cells plug tray ${ }^{-1}$. Amino acids-sprayed plants from 50-cell plug tray ${ }^{-1}$ increased root: shoot ratio, except for the Mix and tryptophansprayed ones. On the other hand, in plants from 288-plug cell tray ${ }^{-1}$ all amino acids treatments increased root: shoot ratio over controls. A similar trend was found when the root: shoot allometries were performed. The higher partition coefficient $(\beta)$ was found in plants from 288-cell plug tray ${ }^{-1}$ and in control plants from both cell volume treatment (Table 4).

Despite the technological use of plug cell trays in vegetable industry, they imply the presence of an abiotic root restriction stress (Di 
Benedetto, 2011). To override these effects on plant growth, the use of different bio stimulants commercial formulations has been proposed (Rai, 2002; Shehata et al., 2011, 2016; Colla et al., 2015; Häusler et al., 2014). However, the most physiological mechanism involved has not been completely documented. Biostimulant meaning included all substance or microorganism exogenously supplied to plants which increased growth, environmental stress tolerance or traits related with the commercial quality (Du Jardin, 2015). In this context, the use of amino acids would be justified, although a few data on vegetables are available (Polo et al., 2006; Liu et al., 2008; El-Aal et al., 2010; Haghighi and Barzegar, 2017; Kunicki et al., 2010; Shehata et al., 2011).

Our results showed a higher fresh weight accumulation at the end of the experiment in plants from 50-cells plug trays ${ }^{-1}$ which suggested a lower root restriction level (Table 1) in agreement with previous reports (Williams et al., 2016; Rattin et al., 2017; De Lojo et al., 2017; Geraci et al., 2018). A foliar spray containing all the amino acids tested (Mix solution) when lettuce plants only had expanded the second true leaves pair, decreased fresh weight 120 days later. These results would be explained by the antagonistic effects showed by single amino acids solutions (glutamic acid, cysteine, glycine, leucine and valine) (Table1). On the contrary, when plants from 288-cells plug trays ${ }^{-1}$ were early sprayed with Mix solution, the higher fresh weight accumulation (similar to the controls 50-cells plug trays $^{-1}$ ) was found although with significant differences between single amino acids solutions (Table 1), in agreement with Linker and Johnson-Rutzke (2005) and Mondal et al., (2015).

At plant level, biomass accumulation on a fresh-dry weight is related to total leaf area expanded and photo assimilates both fixation and partitioning (Andrews et al., 2013). The effect of a root restriction related to plug cell volume on lettuce was similar to previously finding on fresh weight (Table 2), although significant differences between single amino acids solutions and controls lettuce increased as well.

Total leaf area is the result of leaf initiation and leaf expansion, estimated through RLA and RLAE respectively (Table 2). Although there is no previous report on the effect of amino acids sprays on total leaf area, our results showed that both growth parameters were higher in lettuce plants from 50-cells plug trays ${ }^{-1}$. However, while RLA increased from amino acids-sprayed plants with both cell plug trays, RLAE only showed significant increases in plants from 288-cells plug trays ${ }^{-1}$.

Higher biomass accumulation on a dry weight basis where found in lettuce plants with the lowest root restrictions (50-cells plug trays ${ }^{-1}$ ) (Figure 1A). However, different amino acids spray showed significant differences regarding controls in plants from 288-cells plug trays ${ }^{-1}$ (Figure 1B), in agreement with Mondal et al., (2015).

Higher RGR and NAR values but lower LAR values from 50-cells plug trays ${ }^{-1}$ than those from 288-cells plug trays ${ }^{-1}$ were found (Table $3)$. On the other hand, the positive NAR (Figure 2A), LAR (Figure 2B) and RGR relationships would indicate that the higher biomass accumulation in some amino acidssprayed lettuce plants would be related with as a direct higher photosynthetic capacity (associated with NAR changes) as leaf structure changes which facilitate $\mathrm{CO}_{2}$ internal mesophyll diffusion (associated with LAR changes) in agreement with Gandolfo et al., (2014). Allometries from roots and shoots let to estimate the photo assimilates partitioning between them. The higher $\beta$ coefficients in lettuce plants from 288-cells plug trays ${ }^{-1}$ 
suggested a higher photo assimilates partitioning to roots. On the other hand, plants from 50 cells plug trays ${ }^{-1}$ and amino acidstreated plants showed a higher photo assimilates partitioning to shoots (Table 4).

These results set out two questions. The first is related to similar responses found in other vegetables (Pagani et al., 2013; Coro et al., 2014; Di Matteo et al., 2015; Della Gaspera et al., 2016; Geraci et al., 2018) and ornamentals (Di Benedetto and Pagani, 2013; De Lojoand Di Benedetto, 2014; Gandolfo et al., 2014; De Lojo et al., 2017; Molinari et al., 2018; Piotti et al., 2018) where growth has been stimulated in cytokinins-treated plants. A nitrogen availability, cytokinins synthesis and root: shoot ratio relationships have been indicated. However, it is unclear how nitrogen availability is linked with changes in specific cytokinins and how these can change photo assimilates allocation. A feasible hypothesis is that both root and shoot relative growth rates can be modulated by signals related to both carbon and nitrogen status (Grechy et al., 2007). Cambri et al., (2008) and Biancucci et al., (2015) showed that root elongation is promoted by exogenous supply of proline amino acid, suggested it involvement in the response to abiotic stresses. In the same way, Mano et al., (2012) and Halpern et al., (2015) showed a stimulant growth effect by tryptophan, presumably attributable to auxin synthesis increase. There are only 20 amino acids involved in protein building, but there are 250 more that are known involved in different physiological plant mechanisms (Halpern et al., 2015). Although amino acids are a nitrogen source, the usually concentrations involved in exogenous supply are too low that its positive effects can be attributable to an increase in nitrogen availability.

Table.1 Total fresh weight at the end of the experiment in lettuce plants from 50- or 288-cell plug tray-1 and sprayed with thirteen amino acids solutions. Different lower case letters indicate significant differences $(\mathrm{P}<0.05)$ between control and amino acids-sprayed treatments. Different capital letters indicate significant differences $(P<0.05)$ between plants from different cell plug tray-1

\begin{tabular}{|l|c|c|}
\hline & \multicolumn{2}{|c|}{$\begin{array}{c}\text { Fresh weight } \\
\text { (g plant }\end{array}$} \\
\hline & 50 -cells & 288 -cells \\
\hline Control & & \\
\hline Mix & $62.83 \mathrm{bA}$ & $50.27 \mathrm{bB}$ \\
\hline Aspartic acid & $54.48 \mathrm{cB}$ & $61.00 \mathrm{aA}$ \\
\hline Glutamic acid & $60.42 \mathrm{bA}$ & $35.39 \mathrm{~dB}$ \\
\hline Alanine & $55.67 \mathrm{cA}$ & $49.00 \mathrm{bB}$ \\
\hline Cysteine & $60.47 \mathrm{bA}$ & $44.75 \mathrm{cB}$ \\
\hline Glycine & $49.88 \mathrm{dA}$ & $40.56 \mathrm{cB}$ \\
\hline Leucine & $44.22 \mathrm{dA}$ & $31.46 \mathrm{~dB}$ \\
\hline Lysine & $56.47 \mathrm{cA}$ & $49.83 \mathrm{bB}$ \\
\hline Methionine & $67.50 \mathrm{aA}$ & $49.92 \mathrm{bB}$ \\
\hline Threonine & $59.61 \mathrm{bA}$ & $41.91 \mathrm{cB}$ \\
\hline Tryptophan & $62.15 \mathrm{bA}$ & $52.71 \mathrm{bB}$ \\
\hline Valine & $66.93 \mathrm{aA}$ & $48.10 \mathrm{bB}$ \\
\hline & $57.01 \mathrm{cA}$ & $43.98 \mathrm{cB}$ \\
\hline
\end{tabular}


Table.2 Changes in total leaf area, RLAE, RLA and SLA during the experiment for lettuce plants grown in 50- or 288-cell plug tray-1 and sprayed with thirteen amino acids solutions. Different lower case letters indicate significant differences $(\mathrm{P}<0.05)$ between control and amino acids-sprayed treatments. Different capital letters indicate significant differences $(\mathrm{P}<0.05)$ between plants from different cell plug tray-1. The probability of the slope being zero for RLAE and RLA was $\mathrm{P}<.001$

\begin{tabular}{|c|c|c|c|c|c|c|c|c|}
\hline & \multicolumn{2}{|c|}{$\begin{array}{c}\text { Leaf area } \\
\left(\mathrm{cm}^{2} \text { plant }^{-1}\right)\end{array}$} & \multicolumn{2}{|c|}{$\begin{array}{c}\text { RLAE } \\
\left(\mathrm{cm}^{2} \mathrm{~cm}^{-2} \text { day }^{-1}\right)\end{array}$} & \multicolumn{2}{|c|}{$\begin{array}{c}\text { RLA } \\
\text { (leaves week }{ }^{-1} \text { plant }^{-1} \text { ) }\end{array}$} & \multicolumn{2}{|c|}{$\underset{\left(\mathrm{cm}^{2} \mathbf{g}^{-1}\right)}{\operatorname{SLA}}$} \\
\hline & 50 -cells & 288-cells & 50-cells & 288-cells & 50-cells & 288-cells & 50-cells & 288-cells \\
\hline Control & $1654.05 \mathrm{bA}$ & $1532.88 \mathrm{bB}$ & $0.0850 \mathrm{cA}$ & $0.0766 \mathrm{cB}$ & $0.2458 \mathrm{cA}$ & $0.2169 \mathrm{hB}$ & $35.11 \mathrm{cA}$ & $36.50 \mathrm{bA}$ \\
\hline Mix & $1546.27 \mathrm{cB}$ & $1739.70 \mathrm{aA}$ & $0.0840 \mathrm{cB}$ & $0.0879 \mathrm{aA}$ & $0.2511 \mathrm{bB}$ & $0.3053 \mathrm{aA}$ & $40.78 \mathrm{bA}$ & $32.40 \mathrm{cB}$ \\
\hline Aspartic acid & $1180.00 \mathrm{fA}$ & $1184.56 \mathrm{eA}$ & $0.0918 \mathrm{aA}$ & $0.0747 \mathrm{cB}$ & $0.2213 \mathrm{fA}$ & $0.1973 \mathrm{iB}$ & $25.70 \mathrm{~dB}$ & $35.86 \mathrm{bA}$ \\
\hline Glutamic acid & $1429.57 \mathrm{dA}$ & $1358.73 \mathrm{cA}$ & $0.0936 \mathrm{aA}$ & $0.0771 \mathrm{cB}$ & $0.2222 \mathrm{fB}$ & $0.2733 \mathrm{bA}$ & $35.26 \mathrm{cA}$ & $36.88 \mathrm{bA}$ \\
\hline Alanine & $1816.43 \mathrm{aA}$ & $1198.20 \mathrm{eB}$ & $0.0904 \mathrm{abA}$ & $0.0800 \mathrm{bB}$ & $0.2813 \mathrm{aA}$ & $0.1484 \mathrm{kB}$ & $43.26 \mathrm{aA}$ & $37.11 \mathrm{bB}$ \\
\hline Cysteine & $1564.78 \mathrm{cA}$ & $1238.90 \mathrm{~dB}$ & $0.0896 \mathrm{bA}$ & $0.0739 \mathrm{cB}$ & $0.2347 \mathrm{eB}$ & $0.2476 \mathrm{eA}$ & $32.56 \mathrm{cB}$ & $37.08 \mathrm{bA}$ \\
\hline Glycine & $1376.25 \mathrm{eA}$ & $1002.66 \mathrm{fB}$ & $0.0878 \mathrm{bA}$ & $0.0699 \mathrm{~dB}$ & $0.2400 \mathrm{dA}$ & $0.1707 \mathrm{jB}$ & $35.68 \mathrm{cA}$ & $33.81 \mathrm{bA}$ \\
\hline Leucine & $1439.36 \mathrm{dA}$ & $1318.80 \mathrm{cB}$ & $0.0890 \mathrm{bA}$ & $0.0789 \mathrm{bB}$ & $0.2578 \mathrm{bA}$ & $0.2329 \mathrm{gB}$ & $35.83 \mathrm{cA}$ & $35.78 \mathrm{bA}$ \\
\hline Lysine & $1868.13 \mathrm{aA}$ & $1284.00 \mathrm{~dB}$ & $0.0904 \mathrm{abA}$ & $0.0774 \mathrm{cB}$ & $0.2524 \mathrm{bA}$ & $0.2422 \mathrm{fB}$ & $38.10 \mathrm{bA}$ & $33.58 \mathrm{bB}$ \\
\hline Methionine & $1656.82 \mathrm{bA}$ & $1365.91 \mathrm{cB}$ & $0.0917 \mathrm{aA}$ & $0.0769 \mathrm{cb}$ & $0.2529 \mathrm{bA}$ & $0.2573 \mathrm{dA}$ & $33.25 \mathrm{cA}$ & $33.52 \mathrm{bA}$ \\
\hline Threonine & $1675.36 \mathrm{bA}$ & $1420.34 \mathrm{bB}$ & $0.0901 \mathrm{abA}$ & $0.0788 \mathrm{bB}$ & $0.2253 \mathrm{fB}$ & $0.2609 \mathrm{cA}$ & $35.90 \mathrm{cB}$ & $43.62 \mathrm{aA}$ \\
\hline Tryptophan & $1596.15 \mathrm{cA}$ & $1323.26 \mathrm{cB}$ & $0.0910 \mathrm{aA}$ & $0.0759 \mathrm{cB}$ & $0.2529 \mathrm{bA}$ & $0.2418 \mathrm{fB}$ & $34.02 \mathrm{cA}$ & $34.41 \mathrm{bA}$ \\
\hline Valine & $1536.38 \mathrm{cA}$ & $1461.25 \mathrm{bB}$ & $0.0933 \mathrm{aA}$ & $0.0727 \mathrm{~dB}$ & $0.2356 \mathrm{eB}$ & $0.2484 \mathrm{eA}$ & $32.49 \mathrm{cA}$ & $33.27 \mathrm{bA}$ \\
\hline
\end{tabular}


Table.3 Changes in RGR, NAR and LAR during the experiment for lettuce plants grown in 50or 288-cell plug tray-1 and sprayed with thirteen amino acids solutions. Different lower case letters indicate significant differences $(\mathrm{P}<0.05)$ between control and amino acids-sprayed treatments. Different capital letters indicate significant differences $(\mathrm{P}<0.05)$ between plants from different cell plug tray-1. The probability of the slope being zero for RGR was $\mathrm{P}<.001$

\begin{tabular}{|c|c|c|c|c|c|c|}
\hline & \multicolumn{2}{|c|}{$\begin{array}{c}\text { RGR } \\
\left(\mathrm{g} \mathrm{g}^{-1} \text { day }^{-1}\right)\end{array}$} & \multicolumn{2}{|c|}{$\begin{array}{c}\text { NAR } \\
\left(\mathrm{g} \mathrm{cm}^{-2} \mathrm{day}^{-1}\right)\left(\mathbf{x ~ 1 0}^{-5}\right)\end{array}$} & \multicolumn{2}{|c|}{$\underset{\left(\mathrm{cm}^{2} \mathbf{g}^{-1}\right)}{\text { LAR }}$} \\
\hline & 50-cells & 288-cells & 50-cells & 288-cells & 50-cells & 288-cells \\
\hline Control & $0.0799 \mathrm{aA}$ & $0.0763 \mathrm{bA}$ & $32.60 \mathrm{cA}$ & $31.11 \mathrm{aA}$ & $245.08 \mathrm{aA}$ & $245.23 \mathrm{aA}$ \\
\hline Mix & $0.0772 \mathrm{aB}$ & $0.0871 \mathrm{aA}$ & $34.38 \mathrm{cA}$ & $30.68 \mathrm{aB}$ & $224.55 \mathrm{bB}$ & $283.92 \mathrm{aA}$ \\
\hline Aspartic acid & $0.0782 \mathrm{aA}$ & $0.0739 \mathrm{bA}$ & $40.18 \mathrm{aA}$ & $29.22 \mathrm{aB}$ & $194.61 \mathrm{bB}$ & $252.93 \mathrm{aA}$ \\
\hline Glutamic acid & $0.0820 \mathrm{aA}$ & $0.0735 \mathrm{bB}$ & $39.27 \mathrm{aA}$ & $30.34 \mathrm{aB}$ & $208.80 \mathrm{bB}$ & $242.23 \mathrm{aA}$ \\
\hline Alanine & $0.0776 \mathrm{aA}$ & $0.0729 \mathrm{bA}$ & $35.30 \mathrm{bA}$ & $29.73 \mathrm{aB}$ & $219.82 \mathrm{bB}$ & $245.18 \mathrm{aA}$ \\
\hline Cysteine & $0.0760 \mathrm{bA}$ & $0.0741 \mathrm{bA}$ & $39.94 \mathrm{aA}$ & $29.02 \mathrm{aB}$ & $190.28 \mathrm{bB}$ & $255.34 \mathrm{aA}$ \\
\hline Glycine & $0.0778 \mathrm{aA}$ & $0.0650 \mathrm{cB}$ & $36.91 \mathrm{bA}$ & $27.66 \mathrm{aB}$ & $210.76 \mathrm{bA}$ & $234.98 \mathrm{aA}$ \\
\hline Leucine & $0.0785 \mathrm{aA}$ & $0.0749 \mathrm{bA}$ & $36.77 \mathrm{bA}$ & $30.56 \mathrm{aB}$ & $213.51 \mathrm{bB}$ & $245.08 \mathrm{aA}$ \\
\hline Lysine & $0.0820 \mathrm{aA}$ & $0.0716 \mathrm{bB}$ & $33.01 \mathrm{cA}$ & $28.13 \mathrm{aB}$ & $248.41 \mathrm{aA}$ & $254.53 \mathrm{aA}$ \\
\hline Methionine & $0.0764 \mathrm{bA}$ & $0.0769 \mathrm{bA}$ & $37.45 \mathrm{bA}$ & $29.19 \mathrm{aB}$ & $204.02 \mathrm{bB}$ & $263.41 \mathrm{aA}$ \\
\hline Threonine & $0.0818 \mathrm{aA}$ & $0.0747 \mathrm{bB}$ & $39.37 \mathrm{aA}$ & $29.84 \mathrm{ab}$ & $207.78 \mathrm{bB}$ & $250.33 \mathrm{aA}$ \\
\hline Tryptophan & $0.0775 \mathrm{aA}$ & $0.0750 \mathrm{bA}$ & $38.29 \mathrm{aA}$ & $27.76 \mathrm{aB}$ & $202.41 \mathrm{bB}$ & $270.16 \mathrm{aA}$ \\
\hline Valine & $0.0799 \mathrm{aA}$ & $0.0747 \mathrm{bA}$ & $35.11 \mathrm{bA}$ & $27.63 \mathrm{aB}$ & $227.58 \mathrm{bB}$ & $270.35 \mathrm{aA}$ \\
\hline
\end{tabular}

Table. 4 Changes in allometric relationships between roots and shoots for lettuce plants grown in 50- or 288-cell plug tray-1 and sprayed with thirteen amino acids solutions. Different lower case letters indicate significant differences $(\mathrm{P}<0.05)$ between control and amino acids-sprayed treatments. Different capital letters indicate significant differences $(\mathrm{P}<0.05)$ between plants from different cell plug tray-1. The probability of the slope being zero for $\beta$ coefficient was $\mathrm{P}<.001$

\begin{tabular}{|l|c|c|c|c|}
\hline & \multicolumn{2}{|c|}{ Root : Shoot ratio } & \multicolumn{2}{c|}{$\boldsymbol{\beta}$} \\
\hline & 50 -cells & 288 -cells & 50 -cells & 288 -cells \\
\hline Control & & & & \\
\hline Mix & $0.195 \mathrm{dA}$ & $0.179 \mathrm{~dB}$ & $0.782 \mathrm{bA}$ & $0.781 \mathrm{aA}$ \\
\hline Aspartic acid & $0.191 \mathrm{dA}$ & $0.202 \mathrm{dA}$ & $0.713 \mathrm{cA}$ & $0.704 \mathrm{dA}$ \\
\hline Glutamic acid & $0.280 \mathrm{cA}$ & $0.303 \mathrm{cA}$ & $0.777 \mathrm{bA}$ & $0.800 \mathrm{aA}$ \\
\hline Alanine & $0.252 \mathrm{cB}$ & $0.245 \mathrm{dA}$ & $0.666 \mathrm{~dB}$ & $0.752 \mathrm{bA}$ \\
\hline Cysteine & $0.241 \mathrm{cB}$ & $0.359 \mathrm{bA}$ & $0.742 \mathrm{cB}$ & $0.825 \mathrm{aA}$ \\
\hline Glycine & $0.256 \mathrm{cA}$ & $0.252 \mathrm{dA}$ & $0.712 \mathrm{cA}$ & $0.660 \mathrm{eB}$ \\
\hline Leucine & $0.269 \mathrm{cB}$ & $0.388 \mathrm{bA}$ & $0.641 \mathrm{~dB}$ & $0.797 \mathrm{aA}$ \\
\hline Lysine & $0.249 \mathrm{cB}$ & $0.319 \mathrm{cA}$ & $0.711 \mathrm{cB}$ & $0.821 \mathrm{aA}$ \\
\hline Methionine & $0.364 \mathrm{bA}$ & $0.297 \mathrm{~dB}$ & $0.750 \mathrm{bB}$ & $0.792 \mathrm{aA}$ \\
\hline Threonine & $0.407 \mathrm{aB}$ & $0.491 \mathrm{aA}$ & $0.773 \mathrm{bB}$ & $0.838 \mathrm{aA}$ \\
\hline Tryptophan & $0.192 \mathrm{dA}$ & $0.242 \mathrm{dA}$ & $0.730 \mathrm{cA}$ & $0.703 \mathrm{dA}$ \\
\hline Valine & $0.438 \mathrm{aA}$ & $0.252 \mathrm{~dB}$ & $0.902 \mathrm{aA}$ & $0.763 \mathrm{bB}$ \\
& & & & \\
\hline
\end{tabular}


Fig.1 Dry weight at the end of the experiment on lettuce plants grown in 50- (A) or 288-cell plug tray-1 (B) and sprayed with thirteen amino acids solutions. Different lower case letters indicate significant differences $(\mathrm{P}<0.05)$ between control and amino acids-sprayed treatments. Vertical lines indicate standard errors

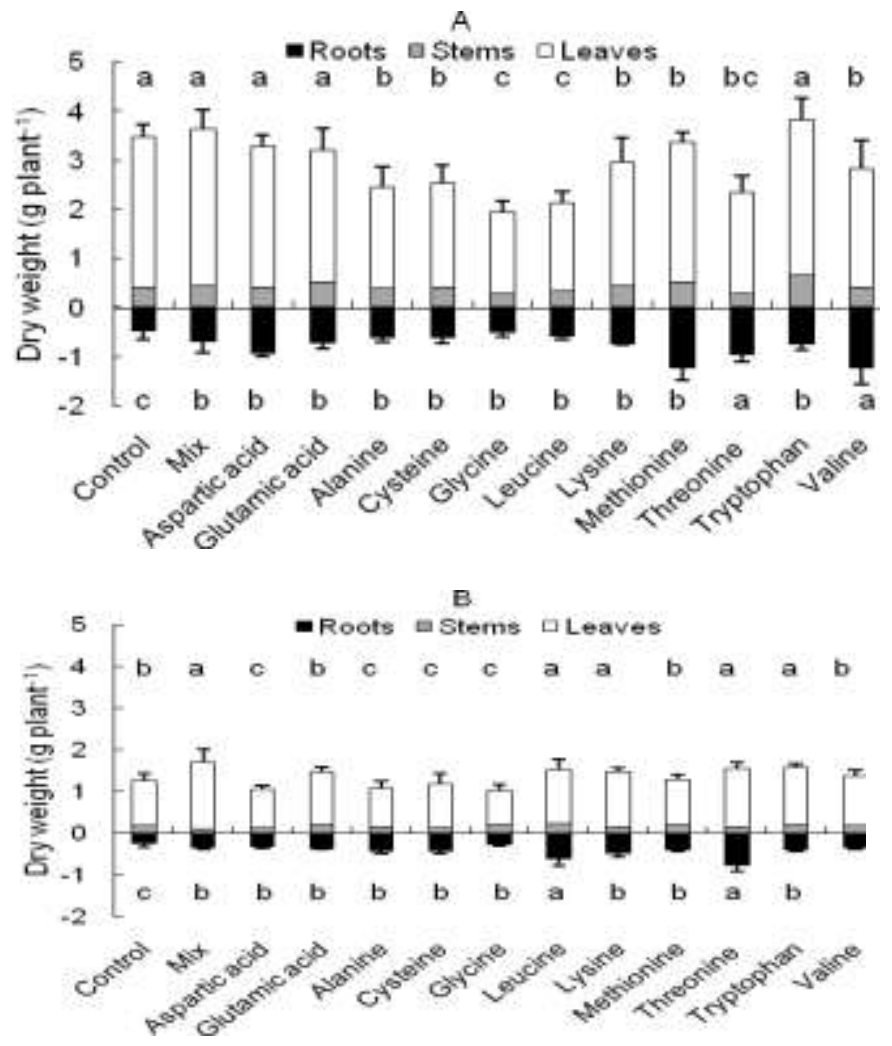

Fig.2 NAR (A) and LAR (B) related to RGR for lettuce plants grown in 50- or 288-cell plug tray-1 and sprayed with thirteen amino acids solutions (fully symbols). Control plants: empty symbols. The straight-line regressions wereNAR50 $=497.00 \mathrm{RGR}-2.02(\mathrm{r} 2=0.653 ; \mathrm{P}<0.05)$; NAR288 = 137.65 RGR + $19.02(\mathrm{r} 2=0.291 ; \mathrm{P}<0.05)$; LAR50 = $4182.30 \mathrm{RGR}-114.50(\mathrm{r} 2=$ $0.648 ; \mathrm{P}<0.05) ; \mathrm{LAR} 288=2195.30 \mathrm{RGR}+91.00(\mathrm{r} 2=0.579 ; \mathrm{P}<0.05)$
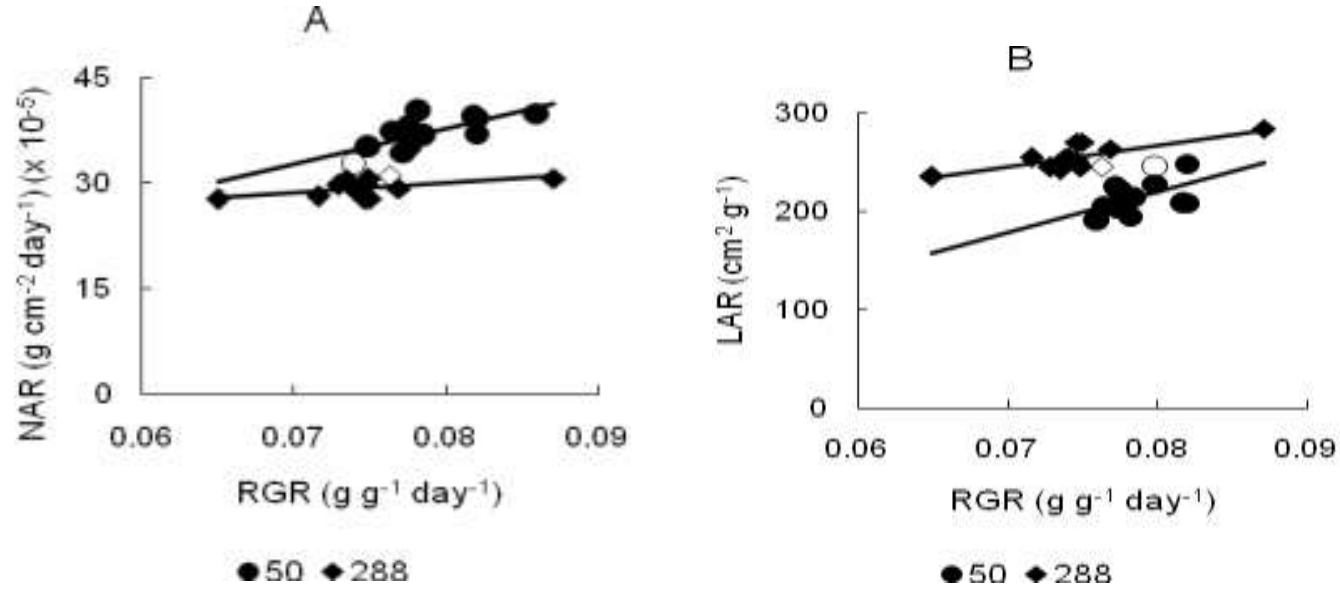
As nitrate is assimilated into conversion to nitrite, ammonia and then into amino acids, enzymes responsible to cytokinin synthesis are specifically induced by nitrate supply (Miyawaki et al., 2004) which was indicated by the fact the higher nitrogen supply the higher cytokinins concentrations (Takei et al., 2002). A similar pathway control of endogenous amino acids has been suggested by Miller et al., (2002). In the same way, Garnica et al., (2010) showed that nitrates have been related to clear increases in the main active forms of cytokinins and reduction of the levels of the lower active forms, independently of the dose applied. Likewise, the presence of nitrate also enhanced indole acetic shoots content, which correlated with higher cytokinin levels (Pavlikova et al., 2012).

A second point, but not of less importance, is the specific effect of each individual amino acid on plant growth, considering that the most commercial formulations usually included a lot of them. A key view to understand plant carbon-nitrogen relationships is the fact that nitrogen assimilation capacity is lined up to both nutrients availability and requirements through an integrated endogenous signals related to hormones, nitrate, sugars, organic acids and amino acids. Responses to nutrient availability changes have a high plasticity degree and require a precise genic coordination (Liu et al., 2009). From a theoretical point of view, it is extremely difficult to predict what specific amino acid can affect some of the many plant physiological mechanisms (Foyer et al., 2003). In this way our results showed that different single amino acids can change different plant traits such as: fresh weight accumulation (Table 1), dry weight accumulation (Figure 1, Table 3), leaf area expansion (Table 1) and photo assimilates partitioning (Table 4). Lea and Miflin (2010) has indicated that the response to an exogenous bio stimulant spray, such as amino acids, is dependent of specie, plant genotype, environment, both formulae concentration and application time. Our present results would be to support it.

In this context, glutamate has been recognized as a signal molecule to plant metabolism (Filleur et al., 2005) and plays a central role in nitrogen metabolism (Schachtman and Shin, 2007). Glutamate has been involved in other minor amino acids metabolism as well. The $\alpha$-amino group of glutamate can be transferred to other amino acids (arginine, proline) by the action of multi specific amino transferases (Forde and Lea, 2007). In the same way, Stitt et al., (2002) have indicated that the amino acid biosynthesis pathways are not limited by the availability of glutamate or other amino donors in central metabolism, at least in plants where the nitrogen supplies are not limiting. However, our results showed that one of the amino acids involved in growth positive responses as control plant as mixed-sprayed plants are glutamic acid.

In summary, a single amino acids spray early on nursery would be a tool for improving lettuce yield in the presence of an abiotic stress related to plug cell volume. The use of 288-cells plug tray would need for more than one single amino acid. Although, additive and antagonistic effects between amino acids would be involved, a conclusive results needs for future experiments which would be included wide amino acids combinations and different plant species.

\section{Acknowledgement}

This work was supported by the University of Buenos Aires Science Program 2017-2020 and the University of Mar del Plata 20182019 Science Program. The authors gratefully acknowledge to Carlos Picone (Aminofert 
Ultra ${ }^{\circledR}$ ) and Alejandro Sala (Evonik Degussa Argentina S.A.) for providing amino acids formulations.

\section{References}

Andrews, M., Raven, J.A., and LeaP.J. 2013. Do plants need nitrate? The mechanisms by which nitrogen form affects plants. Annals of Applied Biology, 163:174-199.

Araki, A., Rattin, J., Di Benedetto, A., and Mirave, P. 2007. Temperature and cytokinin relationships on lettuce (Lactuca sativa L.) and celery (Apium graveolens L.) nursery growth and yield. International Journal of Agricultural Research, 2:725-730.

Biancucci, M., Mattioli, R., Moubayidin, L., Sabatini, S., Costantino, P., and Trovato, M. 2015. Proline affects the size of the root meristematic zone in Arabidopsis. BMC Plant Biology, 15:263.

Cambri, D., Filippini, L., Apone, F.,Arciello, S., Colucci, G., and Portoso, D. 2008. Effect of Aminoplant ${ }^{\circledR}$ on expression of selected genes in Arabidopsis thaliana L. plants. In: Biostimulators in Modern Agriculture. General aspects. (H. Gawronska, ed), Editorial House Wie. Jutra, Varsovia, 77-82.

Colla, G., Nardi, S., Cardarelli, M., Ertani, A., Lucini, L., Canaguier, R., and Rouphael, Y. 2015. Protein hydrolysates as biostimulants in horticulture. Scientia Horticulturae, 196:28-38.

Coro, M., Araki, A., Rattin, J., Miravé, P., and Di Benedetto, A. 2014. Lettuce and celery responses to both $\mathrm{BAP}$ and PBZ related to the plug cell volume. American Journal of Experimental Agriculture, 4: 1103-1119.

Della Gaspera, P., Teruel, J., Giardina, E., and Di Benedetto, A. 2016. Physiological and technological consequences of benzyl adenine (BAP) application on Butternut squash (Cucurbita moschata Duchesne ex Poir.) productivity. American Journal of Experimental Agriculture, 13:1-11.

DeLojo, J., and Di Benedetto, A. 2014. Biomass accumulation and leaf shape can be modulated by an exogenous spray of 6-benzylaminopurine in the ornamental foliage plant Monster adeliciosa (Liebm.). Journal of Horticultural Science \& Biotechnology, 89: 136-140.

DeLojo, J., Gandolfo, E., Gómez, D., Feuring, V., Monti, S., Giardina, E., Boschi, C., and Di Benedetto, A. 2017. Root restriction effects on the bedding pot plant Impatiens walleriana. Journal of Experimental Agriculture International, 15:1-16.

Di Benedetto, A. 2011. Root restriction and post-transplant effects for bedding pot plants. In: Ornamental Plants: Types, Cultivation and Nutrition. (J.C. Aquino, ed.), Nova Science Publishers, Inc. NY, USA. 47-79.

Di Benedetto, A., and Pagani, A. 2013. Dry weight accumulation in the Impatiens walleriana pot plant in responses to different pre-transplant plug cell volume. European Journal of Horticultural Science, 78: 76-85.

Di Mateo, J., Rattin, J., and Di Benedetto, A. 2015. Increase of spinach growth through the use of larger plug cell volume and an exogenous BAP spray. American Journal of Experimental Agriculture, 5:372-383.

Du Jardin, P. 2015. Plant biostimulants: definition, concept, main categories and regulation. Scientia Horticulturae, 196: 3-14.

El-Aal, F.S., Shaheen, A.M., Ahmed, A.A., and Mahmoud, A.R. 2010. Effect of Foliar application of urea and amino 
acids mixtures as antioxidants on growth, yield and characteristics of squash. Research Journal of Agriculture and Biological Sciences, 6:583-588.

Fewtrell, L. 2004. Drinking-water nitrate, methemoglobinemia and global burden of disease: a discussion. Environmental Health Perspective, 112:1371-1374.

Filleur, S., Walch-Liu, P., Gan, Y., and Forde, B.G. 2005. Nitrate and glutamate sensing by plant roots. Biochemical Society Transactions, 33:283-286.

Forde, B.G., and Lea, P.J. 2007. Glutamate in plants: metabolism, regulation, and signaling. Journal of Experimental Botany, 58: 2339-2358.

Foyer, C.H., Parry, M., and Noctor, G. 2003. Markers and signals associated with nitrogen assimilation in higher plants. Journal of Experimental Botany, 54: 585-593.

Gandolfo, E., DeLojo, J., Gómez, D., Pagani, A., Molinari, J., and Di Benedetto, A. 2014.Anatomical changes involved in the response of Impatiens wallerana to different pre-transplant plug cell volumes and BAP sprays. European Journal of Horticultural Science, 79:226-232.

Garnica, M., Houdusse, F., Zamarreno, A.M., and Garcia-Mina, J.M. 2010. The signal effect of nitrate supply enhances active forms of cytokinins and indole acetic content and reduces abscisic acid in wheat plants grown with ammonium. Journal of Plant Physiology, 167:12641272.

Geraci, J., Di Matteo, J., Feuring, V., Giardina, E., and Di Benedetto, A. 2018. Exogenous BAP spray on biomass accumulation in spinach. Journal of Experimental Agriculture International, 25:1- 17.

Grechi, I., Vivin, P., Hilbert, G., Milin, S., Robert, T., and Gaudillere, J.P. 2007. Effect of light and nitrogen supply on internal $\mathrm{C}: \mathrm{N}$ balance and control of root-to-shoot biomass allocation in grapevine. Environmental and Experimental Botany, 59:139-149.

Haghighi, M., and Barzegar, M.R. 2017. Effect of amino acid and mycorrhiza inoculation on sweet pepper growth under greenhouse conditions. Iran Agricultural Research, 36:47-54.

Halpern, M., Bar-Tal, A., Ofek, M., Minz, D., Muller, T., and Yermiyahu, U. 2015. The use of biostimulants for enhancing nutrient uptake. Advances in Agronomy, 130: 141-174.

Häusler, R.E., Ludewig, F., and Krueger, S. 2014. Amino acids-A life between metabolism and signaling. Plant Science, 229:225-237.

He, J., Cheok, L., and Qin, L. 2011. Nitrate accumulation, productivity and photosynthesis of temperate butter head lettuce under different nitrate availabilities and growth irradiances. The Open Horticulture Journal, 4:17-24.

Kunicki, E., Grabowska, A., Sękara, A., and Wojciechowska, R. 2010.The effect of cultivar type, time of cultivation, and biostimulant treatment on the yield of spinach (Spinacia oleracea L.). Folia Horticulturae, 22:9-13.

Lea, P.J., and Miflin, B.J. 2018. Nitrogen assimilation and its relevance to crop improvement. Annual Plant Reviews, 140.

Linker, R., and Johnson-Rutzke, C. 2005. Modeling the effect of abrupt changes in nitrogen availability on lettuce growth, root-shoot partitioning and nitrate concentration. Agricultural Systems, 86:166-189.

Liu, X.Q., Ko, K.Y., Kim, S.H., and Lee, K.S. 2008. Effect of amino acid fertilization on nitrate assimilation of leafy radish and soil chemical properties in high nitrate soil. Communications in Soil Science and Plant Analysis, 39:269-281. 
Liu, T.Y., Chang, C.Y., and Chiou, T.J. 2009. The long-distance signaling of mineral macronutrients. Current Opinion in Plant Biology, 12: 312-319.

Mano, Y., and Nemoto, K. 2012. The pathway of auxin biosynthesis in plants, Journal of Experimental Botany, 63: 2853-2872.

Miller, A.J., Fan, X., Shen, Q., and Smith, S.J. 2007. Amino acids and nitrate as signals for the regulation of nitrogen acquisition. Journal of Experimental Botany, 59:111-119.

Miyawaki, K., Matsumoto-Kitano, M., and Kakimoto, T. 2004. Expression of cytokinin biosynthetic isopentenyl transferase genes in Arabidopsis: tissue specificity and regulation by auxin, cytokinin, and nitrate. The Plant Journal, 37: 128-138.

Molinari, J., Pagani, A., Buyatti, M., Giardina, E., and Di Benedetto, A. 2018. Impatiens 'New Guinea' (Impatiens hawkeri Bull) propagation effects on the pre-transplant biomass accumulation. International Journal of Advances in Agriculture, 3:1-15.

Mondal, M.F., Asaduzzaman, M., Tanaka, H., and Asao, T. 2015. Effects of amino acids on the growth and flowering of Eustoma grandiflorum under autotoxicity in closed hydroponic culture. Scientia Horticulturae, 192:453459.

Pagani, A., Molinari, J., and Di Benedetto, A. 2013. BAP spray and plastic container responses on Asparagus officinalis L. crown growth. Journal of Life Science, 7: 827-835.

Pavlíková, D., Neuberg, M., Žižková, E., Motyka, V., and Pavlík, M. 2012. Interactions between nitrogen nutrition and phytohormone levels in Festulolium plants. Plant Soil and Environment, 58: 367-372.

Piotti, M., Molinari, J., Pagani, A., Giardina,
E., and Di Benedetto, A. 2018. Root restriction effects on Zantedeschia aethiopica. International Journal of Plant \& Soil Science, 23: 1-15.

Polo, J., Barroso, R., Ródenas, J., AzcónBieto, J., Cáceres, R., and Marfà, O. 2006. Porcine hemoglobin hydrolysate as a biostimulant for lettuce plants subjected to conditions of thermal stress. Hort. Technology, 16: 483-487.

Poorter, H., Niklas, K.J., Reich, P.B., Oleksyn, J., Poot, P., and Mommer, L. 2012. Biomass allocation to leaves, stems and roots: meta-analyses of interspecific variation and environmental control. New Phytologist, 193:30-50.

Rai, V.K. 2002. Role of amino acids in plant responses to stresses. Biologia Plantarum, 45:481-487.

Rattin, J., Pico Estrada, O., Giardina, E., and Di Benedetto, A. 2017. Nursery preand post-transplant effects on tomato (Solanum lycopersicum L.) growth and yield. Journal of Experimental Agriculture International, 18:1-14.

Sánchez Andonova, P., Rattin, J., and Di Benedetto, A. 2014. Yield increase as influenced by transplanting of sweet maize (Zea mays L. saccharata). American Journal of Experimental Agriculture, 4: 1314-1329.

Schachtman, D.P., and Shin R. 2007. Nutrient Sensing and Signaling: NPKS. Annual Review of Plant Biology, 58:47-69.

Shehata, S.M., Abdel-Azem, H.S., El-Yazied, A.A., and El-Gizawy, A.M. 2011. Effect of foliar spraying with amino acids and seaweed extract on growth chemical constitutes, yield and its quality of celeriac plant. European Journal of Scientific Research, 58:257265.

Shehata, S.M., Schmidhalter, U., Valšíková, M., and Junge, H. 2016. Effect of biostimulants on yield and quality of head 
lettuce grown under two sources of nitrogen. Gesunde Pflanzen, 68:33-39.

Stagnari, F., Galieni, A., and Pisante, M. 2015. Shading and nitrogen management affect quality, safety and yield of greenhouse-grown leaf lettuce. Scientia Horticulturae, 192:70-79.

Stitt, M., Müller, C., Matt, P., Gibon, Y., Carillo, P., Morcuende, R., Scheible, W., and Krapp, A. 2002. Steps towards an integrated view of nitrogen metabolism. Journal of Experimental Botany, 53:959-970.

Takei, K., Takahashi, T., Sugiyama, T., Yamaya, T., and Sakakibara, H. 2002. Multiple routes communicating nitrogen availability from roots to shoots: a signal transduction pathway mediated by cytokinin. Journal of Experimental Botany, 53:971-977.

Warton, D.I., Duursma, R.A., Falster, D.S., and Taskinen, S. 2012. SMATR 3-an R package for estimation and inference about allometric lines. Methods in Ecology and Evolution, 3: 257-259.

Williams, T., Sala, A., Feuring, V., Giardina, E., and Di Benedetto, A. 2016. Relationships between plug cell size and quality substrate in the bedding pot plant Impatiens wallerana (Hook.f.). International Journal of Plant \& Soil Science, 12: 1-12.

\section{How to cite this article:}

Salinas, M., E. Gandolfo, G. Hakim, E. Giardina and Di Benedetto, A. 2019. Foliar Amino Acids Sprays on Lettuce (Lactuca sativa L.) Biomass Accumulation. Int.J.Curr.Microbiol.App.Sci. 8(01): 2183-2196. doi: https://doi.org/10.20546/ijcmas.2019.801.228 